\title{
Neural Correlates of Face Processing Associated with Language and Social Development in 12-month Infants with Familial Risk of Autism Spectrum Disorder.
}

Joshua Glauser

Harvard University

Carol L Wilkinson ( $\sim$ Carol.Wilkinson@childrens.harvard.edu )

Boston Children's Hospital https://orcid.org/0000-0002-4694-8564

Laurel Joy Gabard-Durnam

Northeastern University

Boin Choi

Boston Children's Hospital

Helen Tager-Flusberg

Boston University

Charles A Nelson

Boston Children's Hospital

\section{Research}

Keywords: EEG, Event Related Potential, Face Processing, Autism, Language Development, Infant

Posted Date: September 1st, 2021

DOl: https://doi.org/10.21203/rs.3.rs-850435/v1

License: (c) (i) This work is licensed under a Creative Commons Attribution 4.0 International License.

Read Full License 


\section{Abstract}

\section{Background}

Identifying neural markers of autism spectrum disorder (ASD) before behavioral symptoms emerge can improve outcomes through early treatment. This study aimed to characterize the neural correlates of face processing in 12-month-old infants at familial risk of developing ASD by (1) comparing face-sensitive event-related potentials (ERP) (Nc, N290, P400) between high-familial-risk infants who develop ASD (HRASD), high-familial-risk infants without ASD (HR-NoASD), and low-familial-risk infants (LR), and (2) evaluating how face-sensitive ERP components are associated with language, communication and social development.

\section{Methods}

12-month-old infants participated in a study in which they were presented with alternating images of their mother's face and the face of a stranger $(L R=45, H R-N o A S D=41, H R-A S D=24)$ as EEG data were collected. Parent-reported and laboratory-observed language and social communication measures were obtained at 12 months, and laboratory-observed social skills measures were conducted at 18 months. Group differences in ERP responses were evaluated using ANOVA, and multiple linear regressions were conducted with maternal education and outcome groups as covariates to assess relationships between ERP and behavioral measures.

\section{Results}

For each of the ERP components (Nc, N290, and P400), the amplitude difference between mother and stranger (Mother-Stranger) trials was not statistically different between the three outcome groups (Nc $p=$ $0.72, \mathrm{~N} 290 \mathrm{p}=0.88, \mathrm{P} 400 \mathrm{p}=0.91$ ). Marginal effects analyses found that within the LR group, a greater Nc Mother-Stranger response was associated with better expressive language skills on the MSEL, controlling for maternal education and outcome group effects (slope 1.15; $p<0.01$ ). No significant associations were observed between the Nc and language or social measures in HR-NoASD or HR-ASD groups. In contrast, specific to the HR-ASD group, amplitude difference between the Mother versus Stranger P400 response was positively associated with expressive (slope $2.1, p<0.001$ ) and receptive language skills at 12-months (slope 1.68, $p<0.005$ ), and better social skills (slope $0.66, p<0.005$ ) at 18 months.

\section{Conclusions}

In 12-month-old infant siblings with subsequent ASD, increased P400 response to Mother over Stranger faces is positively associated with concurrent language and future social skills.

\section{Introduction}


Autism Spectrum Disorder (ASD) is a neurodevelopmental disorder that affects 1 in 54 children in the United States (Maenner, 2020). Symptoms of ASD include deficits in social communication and restrictive/repetitive behaviors that often manifest before the age of three and can persist throughout one's lifetime (Lord et al., 2018). Further, early deficits in social communication can negatively impact the development of social-emotional reciprocity (Rogers et al., 2003), nonverbal communicative behaviors (Kasari et al., 1990), cognitive abilities (Venter et al., 1992), and language development (Cox et al., 1999; Kasari et al., 1990; Sigman et al., 1999). Therefore, early detection and implementation of therapies is crucial to mitigating downstream negative effects of early deficits and promoting effective individualized strategies to support development.

Atypical face processing in individuals with ASD is hypothesized to negatively impact social communication (Høyland et al., 2017), and such differences may be present in infancy, prior to the emergence of behavioral symptoms. To identify such differences, researchers have studied infant siblings of children diagnosed with ASD, as they have an increased incidence of a later ASD diagnosis as well as other developmental delays (Fombonne, 2003; Marrus et al., 2018; Ozonoff et al., 2011; Ritvo et al., 1989). Several eye tracking studies have observed that high familial risk infants show differences in face scanning as early as 6 months of age (eg. eyes vs mouth) and that early differences in attention to faces is associated with later social communication ability (Wagner et al., 2018).

Electrophysiological recordings, and more specifically event-related potentials (ERPs), from infant siblings have also been used to identify neural differences in face processing. There are several ERP components that have been shown to be sensitive to face processing: Nc, N290, and P400. The Nc or 'negative central' waveform is observed over the frontal regions of the brain and is a marker for attention in both infants and adults (Courchesne, 1983). The Nc response is larger in response to novel or unfamiliar objects or faces (Courchesne et al., 1981; Luyster et al., 2011; Nelson, 1994). Across the first 2 years of life, an infant's response to their mother versus a stranger's face shifts, with an increased Nc response to their mother's face before 1 year of age, but a decreased response to their mother versus a stranger by 2 years of age (Carver et al., 2003).

The N290, measured over the lateral-inferior posterior scalp, is the most commonly studied face-sensitive ERP component and is thought to be a precursor to the adult N170 waveform that has robustly been observed in response to faces (Conte et al., 2020; de Haan et al., 2003). The role of the P400 in face processing is less clear, as differential responses to faces in studies have not been consistent (Guy et al., 2016, 2018; Key \& Stone, 2012; McCleery et al., 2009). Given these discrepancies, it has been hypothesized that the P400 may instead play a role in novelty or saliency processing (Conte et al. 2020).

Using a subset of data presented in this paper, our team has previously evaluated ERP responses to mother versus a similarly-looking stranger in high- and low-familial risk infants (Luyster et al., 2011). Overall, no significant risk group differences were observed. At 12 months, both risk groups showed a more negative Nc response to strangers, with the differential response trending larger in the low-risk group. While there was a trend toward higher P400 peak amplitude in the high-risk compared to low-risk 
group, no significant differences were observed in the P400 or N290 component. However, these analyses were performed at the risk group level only, and therefore it is unknown whether there are differences between ASD outcome groups - specifically for those high-risk infants who go on to have autism. In addition, understanding how these face-related ERP components are associated with social and language abilities at the individual level can shed further light on their role in early development.

The current study aims to further investigate differences in ERP response to mother vs stranger and its association with social communication and language skills in low- and high-familial risk infants with and without later ASD diagnosis. First, using a larger data set (102 infants vs 56 infants in Luyster et al 2011) we assessed whether Nc, N290, and P400 responses to mother or stranger faces at 12 months differed between three outcome groups - low risk without ASD (LRC), high-familial-risk without ASD (HR-NoASD), and high-familial-risk with ASD (HR-ASD). Second, we assessed whether 12-month ERP responses to mother or stranger faces were associated with (1) concurrent social communication skills as measured on standardized behavioral measures and parent questionnaires and (2) later social-communication skills as measured by the Autism Diagnostic Observation Schedule (ADOS, (Lord et al., 2000)) Social Affect score and the Communication and Symbolic Behavior Scales (CSBS) Social Composite score at 18 months.

\section{Methods}

\section{Participants}

Infants were enrolled in a longitudinal study conducted jointly by Boston Children's Hospital and Boston University and approved by the institutional review board (\#X06-08-0374). Written consent was obtained from a parent or guardian prior to each child's participation.

Exclusion criteria for the study included prenatal or postnatal medical or neurological problems (e.g. seizures), genetic mutations known to affect neurodevelopment, and uncorrected hearing or visual impairment. All infants had a minimum gestational age of 36 weeks and were from households speaking primarily English (English spoken more than $75 \%$ of the time). Infants were also excluded from this analysis if they did not complete the 12-month visit or complete the ADOS assessment at a later visit.

Infants were enrolled in two groups: (1) high familial risk infants for ASD who had at least one older sibling with ASD, confirmed using the ADOS or the Social Communication Questionnaire (SCQ) (Rutter Bailey \& Lord, 2003), and (2) low risk infants, defined by having a typically developing older sibling, and no first- or second-degree family member with ASD. ASD outcomes groups (LRC, HR-NoASD, and HR-ASD) were determined using behavioral assessments administered at 18-36 month time points (see Behavioral Assessment).

Of the 183 eligible infants who provided EEG data for this analysis, only a subset $(n=110)$ met our behavioral and data quality requirements (Supplemental Figure 1). After ERP preprocessing pipelines 
described below, 102 ERPs were available for Nc analysis (42 LRC, 40 HR-NoASD, 20 HR-ASD) and 64 ERPs were available for N290/P400 analyses (24 LRC, 26 HR-NoASD, 14 HR-ASD).

\section{Behavioral Assessments}

Infants were evaluated using the Mullen Scales of Early Learning (MSEL; Mullen, 1995) at 6, 9, 12, 18, 24, and 36-month visits. These evaluations assessed receptive and expressive language, fine motor skills, and visual reception developmental domains. This study utilizes standardized T-scores from expressive language and receptive language subscales of the MSEL at 12 months of age.

Final ASD outcome groups were determined using the ADOS (Lord et al., 2000), administered at 18, 24, and 36 months of age. For participants receiving an ADOS score indicative of ASD or within 3 points of cutoffs, a licensed clinical psychologist reviewed video recordings of concurrent and previous assessments, and using DSM-5 criteria, provided a best estimate clinical judgment in one of three categories: typically developing, ASD, or non-spectrum disorder (e.g., ADHD, anxiety, language delay). Of the $60 \mathrm{HR}$ infants contributing data for this study, 3 children (2 HR-ASD, 1 HR-No ASD) had final outcome judgements based on only the 18 month ADOS assessment. At 18 months, all participants were administered the ADOS Module 1, and the social affect score was used as one measure of social development.

\section{Parent Questionnaires}

Parents completed the MacArthur-Bates Communicative Development Inventory (MB-CDI): Words and Gestures (Fenson et al., 2007) at the 12-month time point. The study utilizes the Early Gesture and Phrases Understood raw scores from this questionnaire. At the 18-month visit, parents completed the Communication and Symbolic Behavior Scales Developmental Profile (CSBS-DP; Wetherby \& Prizant, 2002). The CSBS-DP is a norm-referenced measure of early social communication and symbolic development. The Social composite standard score (comprised of questions related to emotion, eye gaze, communication, and gestures) was used in subsequent data analyses.

\section{Mother/Stranger Stimuli and EEG Task Procedure}

For this task, infants observe color pictures of their mother and a similarly-looking stranger. Images of the mother and stranger were randomly presented for $500 \mathrm{~ms}$, maintaining a ratio of 1:1 for each type of picture. Pictures of the mothers were matched with strangers according to ethnicity and whether or not they wore glasses. The mothers and strangers had neutral expressions for their pictures.

EEG sessions were conducted in a sound attenuated and electrically shielded room with minimal lighting. During the sessions, caregivers held the infant on their lap, approximately $65 \mathrm{~cm}$ from the experimental monitor. Continuous EEG was recorded using either 64-channel Geodesic Sensor Net 
System or a 128-channel Hydrocel Geodesic Sensor Nets (Electrical Geodesics, Inc., Eugene, OR, USA). Signals were amplified with a Net Amps 200 or Net Amps 300 amplifier (Electrical Geodesic Inc., Eugene, OR, USA), sampled at either $250 \mathrm{~Hz}$ or $500 \mathrm{~Hz}$. EEG data were online-referenced to a single vertex electrode $(\mathrm{Cz})$, and impedances were kept below $100 \mathrm{k} \Omega$. Stimulus presentation was managed via ePrime software (Psychology Software Tools, Pittsburgh, PA). Each stimulus was initiated only when the child was attending to the screen, as observed by an examiner in the adjacent room. Trials during which the child's attention was not maintained on the visual stimulus were marked and then removed from further analysis. A maximum of 100 trials (Mother and Stranger combined) were presented. Fewer trials were presented when the infant became fussy, tired, or inattentive. There was no significant difference in number of trials administered between outcome groups ( $p>0.1$, Supplmental Table 1 ).

\section{EEG Pre-Processing}

The continuous EEG data collected over the mother/stranger paradigm was first downsampled to $250 \mathrm{~Hz}$ in Netstation and then exported to MATLAB (versionR2017b) for preprocessing analysis using a modified version of the Harvard Automated Processing Pipeline for EEG (HAPPE; Gabard-Durnam et al., 2018) to allow for ERP analyses similar to the recently released HAPPE+ER software (Monachino et al., under review). Within the modified HAPPE pipeline, artifact within the continuous EEG data is first extracted using the following steps: a copy of the data is made and that copy is high pass filtered at $1 \mathrm{~Hz}$, channels for subsequent ICA analysis are selected (Supplemental Figure 2), $60 \mathrm{~Hz}$ electrical noise is removed via Cleanline's multi-taper regression (Mullen, 2012), bad channels are rejected, and then remaining artifact is extracted first using wavelet-enhanced independent component analysis (W-ICA), and then subsequently using ICA with MARA automated independent component rejection. Next, the original unfiltered EEG file is subjected to the same channel selection and electrical noise removal steps above and the bad channels detected from analysis on the data copy are removed. The artifact signals identified after the W-ICA step on the data copy are then subtracted from the original unfiltered EEG file, and the identified artifact ICA components rejected from the data copy are back-projected to sensor space as timeseries that are then rejected from the original unfiltered signal. This now 'clean' unfiltered file is filtered using standard ERP filter settings $(0.3 \mathrm{~Hz}-30 \mathrm{~Hz})$, and segmented $(-100 \mathrm{~ms}$ to $700 \mathrm{~ms})$ around the visual stimulus, and baseline corrected via baseline subtraction. Segments with retained artifact in the subset of electrodes used for ERP analyses (Figure 1A and B) are rejected using HAPPE's amplitude (amplitude threshold of $\pm 80 \mu \mathrm{V}$ ) and joint probability criteria, bad channels are interpolated, and data is referenced to the average reference. _

\section{EEG Rejection Criteria}

Children were excluded from the final sample if they had fewer than 10 trials for either the mother or stranger stimuli, or did not meet the following HAPPE data quality output parameters previously determined in this dataset (Wilkinson et al. 2020): percent good channels $>82 \%$, percent of independent 
components rejected $<84 \%$, percent variance of data retained after artifact removal $>32 \%$, mean retained artifact probability $<0.3$. There were no significant differences in data quality between outcome groups. Supplemental Table 1 shows quality metrics for all outcome groups for both ERP analyses.

\section{ERP Analysis}

Average waveforms for each individual participant for each stimulus condition (mother and stranger) were calculated across electrodes in corresponding regions of interest (Nc: Figure 1A, P400: Figure 1B), which were chosen based on previous literature (Guy et al., 2016, 2018; Luyster et al., 2011, 2014). To control for the effect of preceding peak/trough amplitude on the Nc, N290, and P400 amplitudes, all peak amplitudes were calculated by measuring the peak-to-peak amplitude (Richards 2003) - the magnitude of the component value subtracted from the maximum value of the previous opposite polarity peak (Supplemental Figure 3).

For the Nc waveform, the peak negative Nc component was identified as the most negative point between 300 and $600 \mathrm{~ms}$ after the stimulus. The peak negative $\mathrm{N} 290$ components were identified as the most negative point between 200 and 350 ms after the stimulus. The peak positive P400 components was identified as the most positive point between 300 and $500 \mathrm{~ms}$.

To evaluate the difference in response for mother against stranger (Mother-Stranger), for each component, the peak amplitude response to stranger was subtracted from the peak amplitude response to mother.

\section{Statistics}

Demographics were analyzed across groups using Fischer's Exact Test to determine any differences between groups. Continuous variables (eg. EEG HAPPE metrics, ERP component amplitudes) were analyzed for normality, and Kruskal-Wallis $\mathrm{H}$ tests (one-way nonparametric ANOVA) were used to compare groups when Shapiro-Wilk test was $p<0.05$, followed by post hoc Dunn's tests to examine pairwise comparisons. Bonferroni's correction was used to account for multiple comparisons such that family-wise error rate was set to $a<0.05$. Two-way mixed ANOVA were used to determine the effects of group, picture, and group x picture interaction on ERP peak amplitudes.

Simple and multiple linear regressions were used to determine whether ERP peak amplitudes (Mother Stranger) were associated with social and communication measures. Maternal education and ASD outcome group were included in models as covariates to account for their effects on social and communication measures.

\section{Results}




\section{Sample Description}

Demographic data for each outcome group (LRC, HR-NoASD, and HR-ASD) are shown in Table 1. There was a significant group difference in maternal education, with both the HR-NoASD and HR-ASD having a high proportion of mothers with less than a 4-year college degree. Notably, the majority of participants across groups were white with household incomes above $\$ 75,000$.

\section{Grand Average ERP components across groups}

Grand averaged Nc and N290/P400 responses to mother and stranger stimuli by outcome groups are shown in Figure 1A. The effects of group, stimulus, and group $x$ stimulus interaction on peak-peak amplitude, and latency measures were assessed. No significant main effects or interactions were observed. The distribution of Mother-minus-Stranger peak-peak amplitude (Mother-Stranger) across outcome groups is shown in Figure 1B.

\section{ERP Mother-Stranger responses and Communication/Social Measures}

While there were no group differences observed at 12 months of age in N290, P400, and Nc responses to mother/stranger stimuli, there was fairly broad distribution in

responses across infants. We investigated whether an infant's brain response to their mother's versus a stranger's face was associated with concurrent communication or future social skills measures. Scatter plots show the relationship between ERP amplitudes and 12-month communication measures (Figure 2) and 18-month social measures (Figure 3). Across groups, using simple, unadjusted Pearson correlations we observed that increased Nc response to mother over stranger was positively correlated with Expressive Mullen T-scores (Pearson $r=0.32, p=0.0028$ ). Similarly, increased P400 response to mother over stranger was positively correlated with the MB-CDI Phrases Understood.

To further evaluate the effect of group on the relationship between Mother-Stranger ERP responses and communication measures, for each ERP response linear regression models which included two-way interactions between outcome group and the ERP response were examined (Table 2). For all models, maternal education was also included as a covariate. As expected, significant effects of outcome group on MSEL expressive and receptive language scores were observed. For those models where two-way interactions between group and ERP response had a p-value greater than 0.25 , the interaction terms were dropped from the model.

Marginal effects analyses were then performed to further characterize the relationship between ERP responses and communication measures within outcome groups (Table 3). Overall several significant associations, accounting for multiple comparisons, were observed: 
1. Slope comparisons of subgroups from Nc analyses revealed that LR, but not HR-NoASD or HR-ASD infants showed a positive relationship between Nc Mother-Stranger and MSEL Expressive Language tscores (slope $=1.15, p=0.007)$.

2. HR-ASD infants showed a positive relationship between P400 Mother-Stranger response and both MSEL Expressive and Receptive Language t-scores (slope $=2.10, p<0.001$; slope 1.68, $p=0.002$ ).

3. Across groups, P400 Mother-Stranger response was positive associated with the Number of Phrases Understood as measured on the MB-CDI (Adjusted $\mathrm{R}^{2}=0.35 ; \mathrm{p}=0.005$ ).

Similar linear regression models with two-way interactions between outcome group and ERP response were performed for 18-month social measures. Maternal education was again included as a covariate. Summary of models are shown in Supplemental Table 3, and slope comparisons of subgroups are shown in Table 3. Here significant relationships between P400 and social measures were observed specifically for the HR-ASD group. A stronger P400 Mother-Stranger response was associated with better social interaction (lower ADOS Social score and higher CSBS Social score).

\section{Discussion}

Overall, we observed that infants in all three outcome groups had similar ERP responses to pictures of their mother compared to a stranger. The P400 response to mother over stranger was associated with receptive language skills as measured on the MB-CDI. Despite similar ERP responses across groups, we identified outcome group specific relationships between Nc and P400 amplitudes with both communication and social measures. Specifically, for low familial risk infants, Nc was positively associated with expressive language outcomes, whereas, for high familial risk infant with later autism diagnosis, the $\mathrm{P} 400$ was positively associated with concurrent expressive and receptive language development and future social skills.

\section{Lack of differences in ERP amplitudes between cohort groups}

Overall, the three groups presented in the study showed similar Nc, N290, and P400 components to the mother/stranger paradigm at 12 months. For these components, there were no differences in the mother, stranger, or Mother-Stranger amplitude values for any of the groups or between groups. Previous studies in infants have similarly found no significant main effects for familial risk group on N290 and P400 amplitudes in response to faces (Elsabbagh et al., 2009; Luyster et al., 2011; McCleery et al., 2009), but have observed latency differences between risk groups in response to objects. Together, these findings suggest that early face processing is intact in high familial risk infants, including those who later meet ASD criteria. However, studies in preschoolers with ASD have consistently shown differences in ERP responses to familiar/non-familiar faces when compared to typically developing preschoolers (Dawson et al., 2002, 2004). It has been hypothesized that infants with ASD may have delayed development of 
familiar/unfamiliar ERP responses, which may not be captured at a single 12-month time point. Visually, when examining grand averages, we do observe specifically in the LRC group, a downward shift in the frontally measured ERP response to mother, compared to stranger. While these differences were not statistically different, they do suggest a trend in differential responses, and it was this group where we observed a significant association between Nc response and language skills.

\section{Relationships between ERP amplitudes and communication and social measures}

Importantly, this study also investigated whether ERP responses were associated with language and social development, and whether such brain-behavior associations were different between outcome groups. Here we uncovered several interesting findings. First, we observed that for low-familial risk infants, a larger Nc to mother over a stranger was positively associated with concurrent expressive language scores on the MSEL. The Nc amplitude has been observed to change from infancy to preschool years, where in infants under 1 year of age, a more negative response is observed in response to familiar faces; On the other hand, by 3-5 years of age, a more negative response is observed in responses to unfamiliar faces (de Haan et al., 2003; Luyster et al., 2011). Visually the grand average waveforms for the LRC group (Figure 1, top), do show a trend toward increased negative response to mothers, perhaps suggesting that a subset of these infants are making this developmental transition sooner than others. Further, our brain-behavior association suggests that early transition of the Nc's differential familiar/unfamiliar response is associated with more advanced expressive language development. We also note that a similar positive association was observed in the HR-ASD group but was not significant after adjustment for multiple comparisons, likely due to the small sample size within this group. Together, this suggests that Nc response in infancy may not be different between ASD outcomes, but may be an indicator of brain development as it specifically relates to expressive language. Notably, associations were not observed with receptive language and social communication measures. As a form of communication, it is feasible to assume that attentional resources towards a person is more crucial for one's active communication, being expressive, compared to one's passive communication, being receptive. However, since gestures are a precursor to expressive language, it is unclear why gestures would not be similarly significant. More research will be needed to explain these discrepancies in infant attentional resources to their communication and social outcomes.

Second, we observed that the P400 component is significantly associated with MB-CDI Phrases Understood while accounting for maternal education and group, indicating a strong association to receptive language development. In addition, for high-familial risk infants who later met criteria for autism, a greater P400 response to mother over stranger was associated with better concurrent receptive and expressive language, as well as future social skills measured at 18-month. Several studies have investigated clinical correlations of ERP responses to face in infancy. Increased P400 and Nc response to infrequently over frequently shown faces has been associated with better cognitive development (Xie et al., 2019). Differential P400 response changes in facial features has been linked to receptive 
language (Key \& Stone, 2012). While the P400 has been shown to be differentially responsive to faces versus objects in infants as young as 6 months (de Haan \& Nelson, 1999), findings have not been consistent (Conte et al., 2020), and it is unclear if the P400 is a face specific ERP. Both the P400 and Nc components are also hypothesized to be neural markers of sustained attention, as amplitudes are increased in response to novel objects (de Haan \& Nelson, 1997; Reynolds \& Richards, 2005; Xie \& Richards, 2016), as well as communicative over non-communicative gestures (Bakker et al., 2015). We hypothesize that a differential P400 response to mother versus stranger represents an infants' recognition of saliency in their mother's face and that this is predictive of language and social development.

Interestingly we did not observe any brain-behavior associations with the N290, which is the most frequently studied face-specific ERP component, and thought to be a precursor for the N170 (Conte et al., 2020; de Haan et al., 2003). This may be related to developmental timing, and future analyses will investigate whether relationships change over the first three years of life.

\section{Limitations}

This study contained several limitations. The sample size for the HR-ASD group was small, and while findings within the HR-ASD group were highly significant, they should be interpreted with caution and will need to be replicated with a larger sample. Additionally, The sample population had substantially higher maternal education than the national average, indicating that the cohort of infants might not be representative of the general population (US Census Bureau, 2016). Furthermore, HR-ASD infants in this particular sample had language development that fell generally in the average range indicating more high functioning individuals in our analysis; therefore, the analysis does not encompass all of the ASD spectrum in terms of language development.

\section{Conclusions And Future Directions}

We found that there was no difference in the Nc, N290, or P400 responses to mother versus stranger across LRC, HR-NoASD, and HR-ASD groups. However, differential mother vs stranger ERP responses in the Nc and P400 were significantly associated with communication and social development, suggesting they could be a useful biomarker of development for high familial risk infants. Future research will require replication in larger datasets. Further analysis of how differential Nc and P400 responses develop over infancy to preschool age across low and high-risk groups will also provide valuable information on differences in brain development as they relate to language and social development.

\section{Abbreviations}

ADOS: Autism Diagnostic Observation Schedule

ANOVA: Analysis of Variance

Page $11 / 23$ 
ASD: Autism Spectrum Disorder

CDI: MacArthur Bates Communicative Development Inventories

CSBS-DP: Communication and Symbolic Behavior Scales Developmental Profile

EEG: Electroencephalogram

ERP: Event Related Potential

HAPPE: Harvard Automated Processing Pipeline for Electroencephalography

HR-ASD: High Risk with Autism Spectrum Disorder

HR-NoASD: High Risk with no Autism Spectrum Disorder

ICA: Independent Component Analysis

LRC: Low Risk Controls

MARA: Multiple Artifact Rejection Algorithm

MSEL: Mullen Scales of Early Learning

SCQ: Social Communication Questionnaire

W-ICA: Wavelet-enhanced Independent Component Analysis

\section{Declarations}

\section{Ethics approval and consent to participate:}

The study was approved by the institutional review board (\#X06-08-0374).

\section{Consent for publication:}

N/A

\section{Availability of data and materials:}

The datasets used and/or analyzed during the current study are available from the corresponding authors on reasonable request. 


\section{Competing Interests:}

The authors declare that they have no competing interests.

\section{Funding:}

Support for this work was provided by the National Institutes of Health (R01-DC010290 to HTF and CAN; R21 DC 08637 to HTF; K23DC07983-01A1 to CLW), Autism Science Foundation (JG, CLW), Autism Speaks (1323 to HTF), Simons Foundation (137186 to CAN).

\section{Author Contributions:}

J. Glauser: Conceptualization; Data curation - lead; Formal analysis; Writing - original draft. C. L. Wilkinson: Conceptualization - lead; Methodology - lead; Writing - original draft . L. Gabard-Durnam: Conceptualization; Investigation; Data curation; Writing - review and editing. B. Choi: Conceptualization; Investigation; Data acquisition. H. Tager-Flusberg: Conceptualization; Funding acquisition; Supervision; Writing - review and editing. C.A. Nelson: Conceptualization; Funding acquisition; Investigation; Project administration - lead; Supervision; Writing - review and editing.

\section{Acknowledgements:}

We thank all the families and staff involved to make this research possible.

\section{References}

1. Bakker M, Kaduk K, Elsner C, Juvrud J, Gustaf Gredebäck. (2015). The neural basis of non-verbal communication-Enhanced processing of perceived give-me gestures in 9-month-old girls. Frontiers in Psychology, 0. https://doi.org/10.3389/fpsyg.2015.00059.

2. Carver LJ, Dawson G, Panagiotides H, Meltzoff AN, McPartland J, Gray J, Munson J. Age-related differences in neural correlates of face recognition during the toddler and preschool years. Dev Psychobiol. 2003;42(2):148-59. https://doi.org/10.1002/dev.10078.

3. Conte S, Richards JE, Guy MW, Xie W, Roberts JE. Face-sensitive brain responses in the first year of life. Neurolmage. 2020;211:116602. https://doi.org/10.1016/j.neuroimage.2020.116602.

4. Courchesne E. Cognitive Components of the Event-Related Brain Potential: Changes Associated with Development (Vol. 10: Elsevier Science \& Technology; 1983. pp. 329-44. https://doi.org/10.1016/S0166-4115(08)62046-4.

5. Courchesne E, Ganz L, Norcia AM. Event-Related Brain Potentials to Human Faces in Infants. Child Dev. 1981;52(3):804-11. https://doi.org/10.2307/1129080. 
6. Cox A, Charman T, Baron-Cohen S, Drew A, Klein K, Baird G, Swettenham J, Wheelwright S. Autism spectrum disorders at 20 and 42 months of age: Stability of clinical and ADI-R diagnosis. J Child Psychol Psychiatry. 1999;40(5):719-32. https://doi.org/10.1017/S002196309900400X.

7. Dawson G, Carver L, Meltzoff AN, Panagiotides H, McPartland J, Webb SJ. Neural Correlates of Face and Object Recognition in Young Children with Autism Spectrum Disorder, Developmental Delay, and Typical Development. Child Dev. 2002;73(3):700-17.

8. Dawson G, Webb SJ, Carver L, Panagiotides H, McPartland J. Young children with autism show atypical brain responses to fearful versus neutral facial expressions of emotion. Developmental Science. 2004;7(3):340-59. https://doi.org/10.1111/j.1467-7687.2004.00352.x.

9. de Haan M, Johnson MH, Halit H. Development of face-sensitive event-related potentials during infancy: A review. International Journal of Psychophysiology: Official Journal of the International Organization of Psychophysiology. 2003;51(1):45-58. https://doi.org/10.1016/s01678760(03)00152-1.

10. de Haan M, Nelson CA. Recognition of the mother's face by six-month-old infants: A neurobehavioral study. Child Dev. 1997;68(2):187-210.

11. de Haan M, Nelson CA. Brain activity differentiates face and object processing in 6-month-old infants. Dev Psychol. 1999;35(4):1113-21. https://doi.org/10.1037/0012-1649.35.4.1113.

12. Elsabbagh M, Volein A, Csibra G, Holmboe K, Garwood H, Tucker L, Krljes S, Baron-Cohen S, Bolton P, Charman T, Baird G, Johnson MH. Neural Correlates of Eye Gaze Processing in the Infant Broader Autism Phenotype. Biol Psychiat. 2009;65(1):31-8. https://doi.org/10.1016/j.biopsych.2008.09.034.

13. Fenson L, Marchman V, Thal D, Dale P, Reznick JS, Bates E. MacArthur-Bates CDI Words and Gestures. Paul H Brookes; 2007.

14. Fombonne E. Epidemiological Surveys of Autism and Other Pervasive Developmental Disorders: An Update. J Autism Dev Disord. 2003;33(4):365-82. https://doi.org/10.1023/A:1025054610557.

15. Gabard-Durnam LJ, Leal M, Wilkinson AS, C. L., \& Levin AR. The Harvard Automated Processing Pipeline for Electroencephalography (HAPPE): Standardized Processing Software for Developmental and High-Artifact Data. Frontiers in Neuroscience. 2018. https://doi.org/10.3389/fnins.2018.00097.

16. Guy MW, Richards JE, Tonnsen BL, Roberts JE. Neural correlates of face processing in etiologicallydistinct 12-month-old infants at high-risk of autism spectrum disorder. Dev Cogn Neurosci. 2018;29:61-71. https://doi.org/10.1016/j.dcn.2017.03.002.

17. Guy MW, Zieber N, Richards JE. The Cortical Development of Specialized Face Processing in Infancy. Child Dev. 2016;87(5):1581-600. https://doi.org/10.1111/cdev.12543.

18. Høyland AL, Nærland T, Engstrøm M, Lydersen S, Andreassen OA. The relation between face-emotion recognition and social function in adolescents with autism spectrum disorders: A case control study. PLOS ONE. 2017;12(10):e0186124. https://doi.org/10.1371/journal.pone.0186124.

19. Kasari C, Sigman M, Mundy P, Yirmiya N. Affective Sharing In the Context of Joint Attention Interactions of Normal, Autistic, and Mentally-Retarded Children. J Autism Dev Disord. 1990;20(1):87-100. https://doi.org/10.1007/BF02206859. 
20. Key APF, Stone WL. Same but Different: 9-Month-Old Infants at Average and High Risk for Autism Look at the Same Facial Features but Process Them Using Different Brain Mechanisms. Autism Res. 2012;5(4):253-66. https://doi.org/10.1002/aur.1231.

21. Lord C, Elsabbagh M, Baird G, Veenstra-Vanderweele J. Autism spectrum disorder. The Lancet. 2018;392(10146):508-20. https://doi.org/10.1016/S0140-6736(18)31129-2.

22. Lord C, Risi S, Lambrecht L, Cook EH Jr, Leventhal BL, DiLavore PC, Pickles A, Rutter M. The Autism Diagnostic Observation Schedule-Generic: A Standard Measure of Social and Communication Deficits Associated with the Spectrum of Autism. J Autism Dev Disord. 2000;30(3):205-23. https://doi.org/10.1023/A:1005592401947.

23. Luyster R, Powell C, Tager-Flusberg H, Nelson CA. Neural measures of social attention across the first years of life: Characterizing typical development and markers of autism risk. Dev Cogn Neurosci. 2014;8(C):131-43. https://doi.org/10.1016/j.dcn.2013.09.006.

24. Luyster R, Wagner J, Vogel-Farley V, Tager-Flusberg H, Nelson III, C. Neural Correlates of Familiar and Unfamiliar Face Processing in Infants at Risk for Autism Spectrum Disorders. Brain Topogr. 2011;24(3-4):220-8. https://doi.org/10.1007/s10548-011-0176-z.

25. Maenner MJ. (2020). Prevalence of Autism Spectrum Disorder Among Children Aged 8 YearsAutism and Developmental Disabilities Monitoring Network, 11 Sites, United States, 2016. MMWR. Surveillance Summaries, 69. https://doi.org/10.15585/mmwr.ss6904a1.

26. Marrus N, Hall LP, Paterson SJ, Elison JT, Wolff JJ, Swanson MR, Parish-Morris J, Eggebrecht AT, Pruett JR, Hazlett HC, Zwaigenbaum L, Dager S, Estes AM, Schultz RT, Botteron KN, Piven J, Constantino JN, Piven J, Hazlett HC. ... for the IBIS Network. (2018). Language delay aggregates in toddler siblings of children with autism spectrum disorder. Journal of Neurodevelopmental Disorders, 10(1), 29. https://doi.org/10.1186/s11689-018-9247-8.

27. McCleery JP, Akshoomoff N, Dobkins KR, Carver LJ. Atypical Face Versus Object Processing and Hemispheric Asymmetries in 10-Month-Old Infants at Risk for Autism. Biol Psychiat. 2009;66(10):950-7. https://doi.org/10.1016/j.biopsych.2009.07.031.

28. Mullen E. Mullen Scales of Early Learning -AGS Edition Record Form. NCS Pearson; 1995.

29. Nelson CA. Neural correlates of recognition memory in the first postnatal year. In: Human behavior and the developing brain. The Guilford Press; 1994. pp. 269-313.

30. Ozonoff S, Young GS, Carter A, Messinger D, Yirmiya N, Zwaigenbaum L, Bryson S, Carver LJ, Constantino JN, Dobkins K, Hutman T, Iverson JM, Landa R, Rogers SJ, Sigman M, Stone WL. Recurrence Risk for Autism Spectrum Disorders: A Baby Siblings Research Consortium Study. Pediatrics. 2011;128(3):e488-95. https://doi.org/10.1542/peds.2010-2825.

31. Reynolds GD, Richards JE. Familiarization, attention, and recognition memory in infancy: An eventrelated potential and cortical source localization study. Dev Psychol. 2005;41(4):598-615. https://doi.org/10.1037/0012-1649.41.4.598.

32. Ritvo ER, Freeman BJ, Pingree C, Mason-Brothers A, Jorde L, Jenson WR, McMahon WM, Petersen PB, Mo A, Ritvo A. The UCLA-University of Utah epidemiologic survey of autism: Prevalence. The 
American Journal of Psychiatry. 1989;146(2):194-9. https://doi.org/10.1176/ajp.146.2.194.

33. Rogers SJ, Hepburn SL, Stackhouse T, Wehner E. Imitation performance in toddlers with autism and those with other developmental disorders. J Child Psychol Psychiatry. 2003;44(5):763-81. https://doi.org/10.1111/1469-7610.00162.

34. Rutter Bailey A, Lord CM. Social Communication Questionnaire. Los Angeles: Western Pyschological Services; 2003.

35. Sigman M, Ruskin E, Arbelle S, Mervis CB, Byron F, Robinson. Continuity and change in the social competence of children with autism, Down syndrome, and developmental delays. Blackwell Publishers; 1999.

36. US Census Bureau. (2016). Educational Attainment in the United States: 2015. 12.

37. Venter A, Lord C, Schopler E. A Follow-Up Study of High-Functioning Autistic Children. J Child Psychol Psychiatry. 1992;33(3):489-597. https://doi.org/10.1111/j.1469-7610.1992.tb00887.x.

38. Wagner JB, Luyster RJ, Moustapha H, Tager-Flusberg H, Nelson CA. Differential attention to faces in infant siblings of children with autism spectrum disorder and associations with later social and language ability. Int J Behav Dev. 2018. https://doi.org/10.1177/0165025416673475.

39. Wetherby A, Prizant B. CSBS-DP Manual. Paul H Brookes; 2002.

40. Wilkinson CL, Gabard-Durnam LJ, Kapur K, Tager-Flusberg HT, Levin AR, Nelson CA. Use of longitudinal EEG measures in estimating language development in infants with and without familial risk for autism spectrum disorder. Neurobiology of Language. 2020;1(1):33-53.

https://doi:10.1162/nol_a_00002.

41. Xie W, Kumar S, Kakon SH, Haque R, Petri WA, Nelson CA. Chronic inflammation is associated with neural responses to faces in bangladeshi children. Neurolmage. 2019;202:116110-0. https://doi.org/10.1016/j.neuroimage.2019.116110.

42. Xie W, Richards JE. Effects of interstimulus intervals on behavioral, heart rate, and event-related potential indices of infant engagement and sustained attention. Psychophysiology. 2016;53(8):1128-42. https://doi.org/10.1111/psyp.12670.

\section{Tables}

\section{Table 1}

\section{Sample Characteristics}




\begin{tabular}{|c|c|c|c|c|}
\hline & LR & HR-NoASD & HR-ASD & Fisher's exact test \\
\hline & $N=45$ & $N=41$ & $N=24$ & $P$ value \\
\hline Sex & $20 \mathrm{M}, 25 \mathrm{~F}$ & $19 \mathrm{M}, 22 \mathrm{~F}$ & $15 \mathrm{M}, 9 \mathrm{~F}$ & 0.326 \\
\hline Maternal Education, $n$ (\%) & & & & 0.047 \\
\hline Not answered & $5(11)$ & $4(10)$ & $4(17)$ & \\
\hline$<4$-year college degree & $1(2)$ & $7(17)$ & $3(13)$ & \\
\hline 4-year college degree & $8(18)$ & $8(20)$ & $8(33)$ & \\
\hline$>4$-year college degree & $31(69)$ & $22(54)$ & $9(38)$ & \\
\hline Household income, $n$ (\%) & & & & 0.81 \\
\hline Not answered & $8(18)$ & $5(12)$ & $5(21)$ & \\
\hline$<\$ 75,000$ & $6(13)$ & $6(15)$ & $2(8)$ & \\
\hline$>\$ 75,000$ & $31(69)$ & $30(73)$ & $17(71)$ & \\
\hline Race, $n(\%)$ & & & & 0.13 \\
\hline Non-White & $6(13)$ & $3(7)$ & $6(25)$ & \\
\hline
\end{tabular}

\section{Table 2}

Marginal Effects ERP components vs 12-month Language Measures 


\begin{tabular}{|c|c|c|c|c|c|c|c|c|c|}
\hline & & \multicolumn{2}{|c|}{$\begin{array}{l}\text { MSEL } \\
\text { Expressive }\end{array}$} & \multicolumn{2}{|c|}{$\begin{array}{l}\text { MSEL } \\
\text { Receptive }\end{array}$} & \multicolumn{2}{|c|}{$\begin{array}{l}\text { Phrases } \\
\text { Understood }\end{array}$} & \multicolumn{2}{|c|}{ Early Gestures } \\
\hline & & Slope & $P$ value & Slope & $\begin{array}{l}P \\
\text { value }\end{array}$ & Slope & $P$ value & Slope & $\begin{array}{l}\mathrm{P} \\
\text { value }\end{array}$ \\
\hline \multirow[t]{3}{*}{ Nc } & LR & 1.15 & $0.007 *$ & 0.63 & 0.14 & 0.21 & 0.59 & 0.22 & 0.27 \\
\hline & $\begin{array}{l}\text { HR- } \\
\text { noASD }\end{array}$ & 0.35 & 0.46 & -0.25 & 0.61 & 0.33 & 0.35 & 0.05 & 0.80 \\
\hline & HR-ASD & 1.4 & 0.04 & 1.03 & 0.15 & 1.14 & 0.05 & 0.70 & 0.02 \\
\hline \multirow[t]{3}{*}{ P400 } & LR & 0.21 & 0.54 & 0.01 & 0.98 & & & -0.23 & 0.40 \\
\hline & $\begin{array}{l}\text { HR- } \\
\text { noASD }\end{array}$ & 0.03 & 0.94 & 0.06 & 0.88 & & & 0.12 & 0.55 \\
\hline & HR-ASD & 2.10 & $<0.001 *$ & 1.68 & $0.002 *$ & & & 0.60 & 0.02 \\
\hline \multirow[t]{3}{*}{ N290 } & & & & & & & & -0.13 & 0.61 \\
\hline & & & & & & & & 0.15 & 0.27 \\
\hline & & & & & & & & 0.58 & 0.11 \\
\hline
\end{tabular}

Table 3

ERP components vs 18-month Social Measures 


\begin{tabular}{|c|c|c|c|c|c|}
\hline & & \multicolumn{2}{|c|}{ ADOS Social } & \multicolumn{2}{|c|}{ CSBS Social } \\
\hline & & Slope & $P$ value & Slope & $P$ value \\
\hline \multirow[t]{3}{*}{ Nc } & LR & -0.009 & 0.96 & & \\
\hline & HR-noASD & 0.27 & 0.12 & & \\
\hline & HR-ASD & -0.57 & 0.02 & & \\
\hline \multirow[t]{3}{*}{ P400 } & LR & -0.01 & 0.93 & 0.03 & 0.78 \\
\hline & HR-noASD & 0.12 & 0.47 & 0.18 & 0.23 \\
\hline & HR-ASD & -1.22 & $<0.001 *$ & 0.66 & $0.003^{*}$ \\
\hline \multirow[t]{3}{*}{ N290 } & LR & -.12 & 0.59 & & \\
\hline & HR-noASD & -0.12 & 0.58 & & \\
\hline & HR-ASD & -1.03 & 0.02 & & \\
\hline
\end{tabular}

Figures 
A
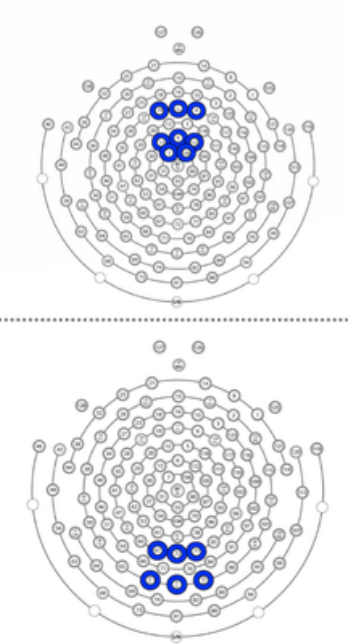

Low Risk

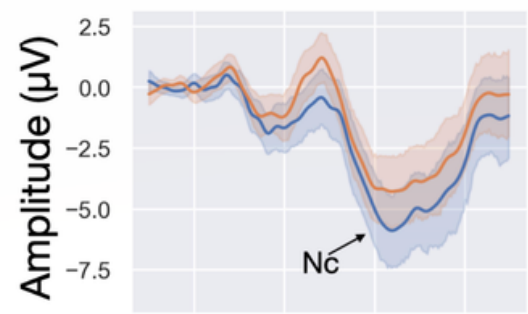

High Risk/No Autism

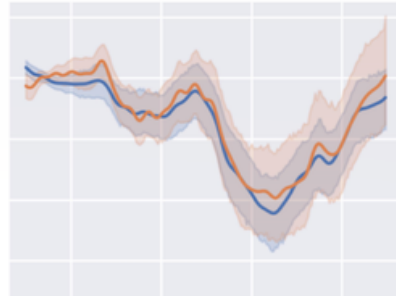

High Risk/Autism
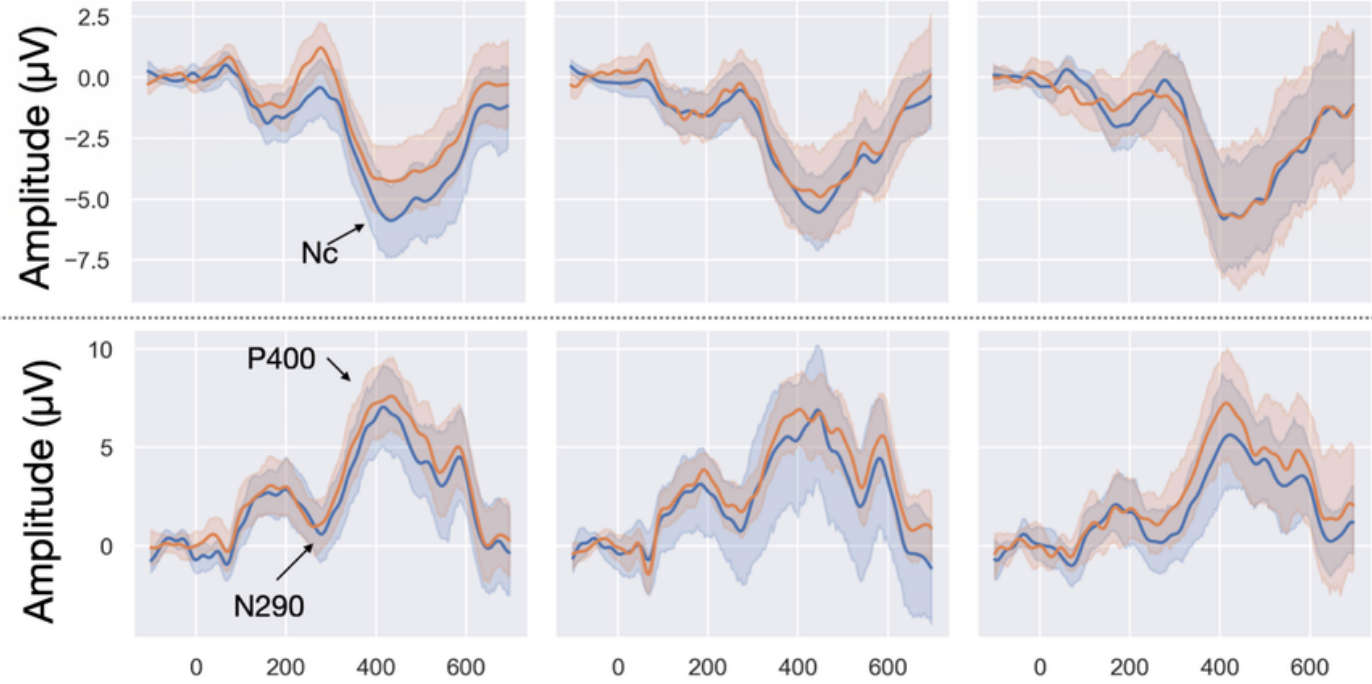

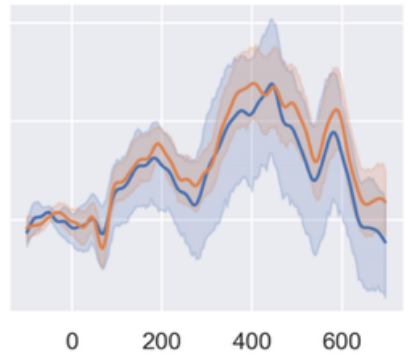

Time (ms)

- Mother - Stranger
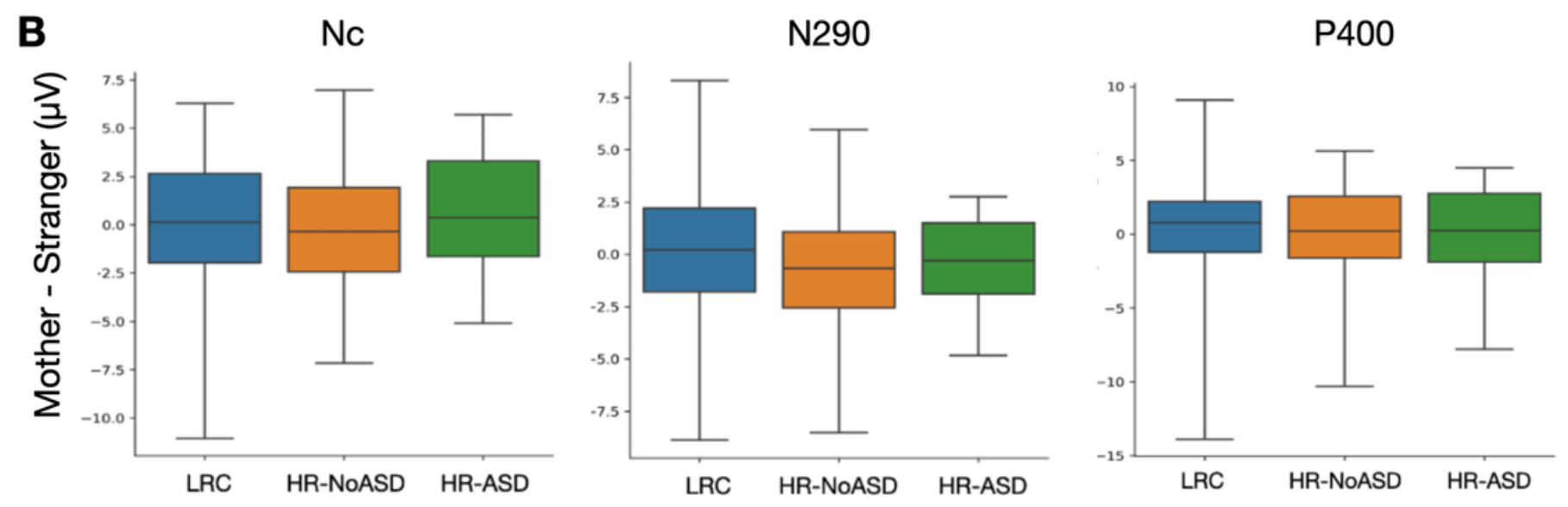

\section{Figure 1}

1A: Grand average ERP waveform across group in response to mother versus stranger faces. Electrode groups and ERP response for Nc (top row) and N290/P400 (bottom row). 1B: Difference in ERP response to mother versus stranger across outcome groups. No significant differences were observed. 


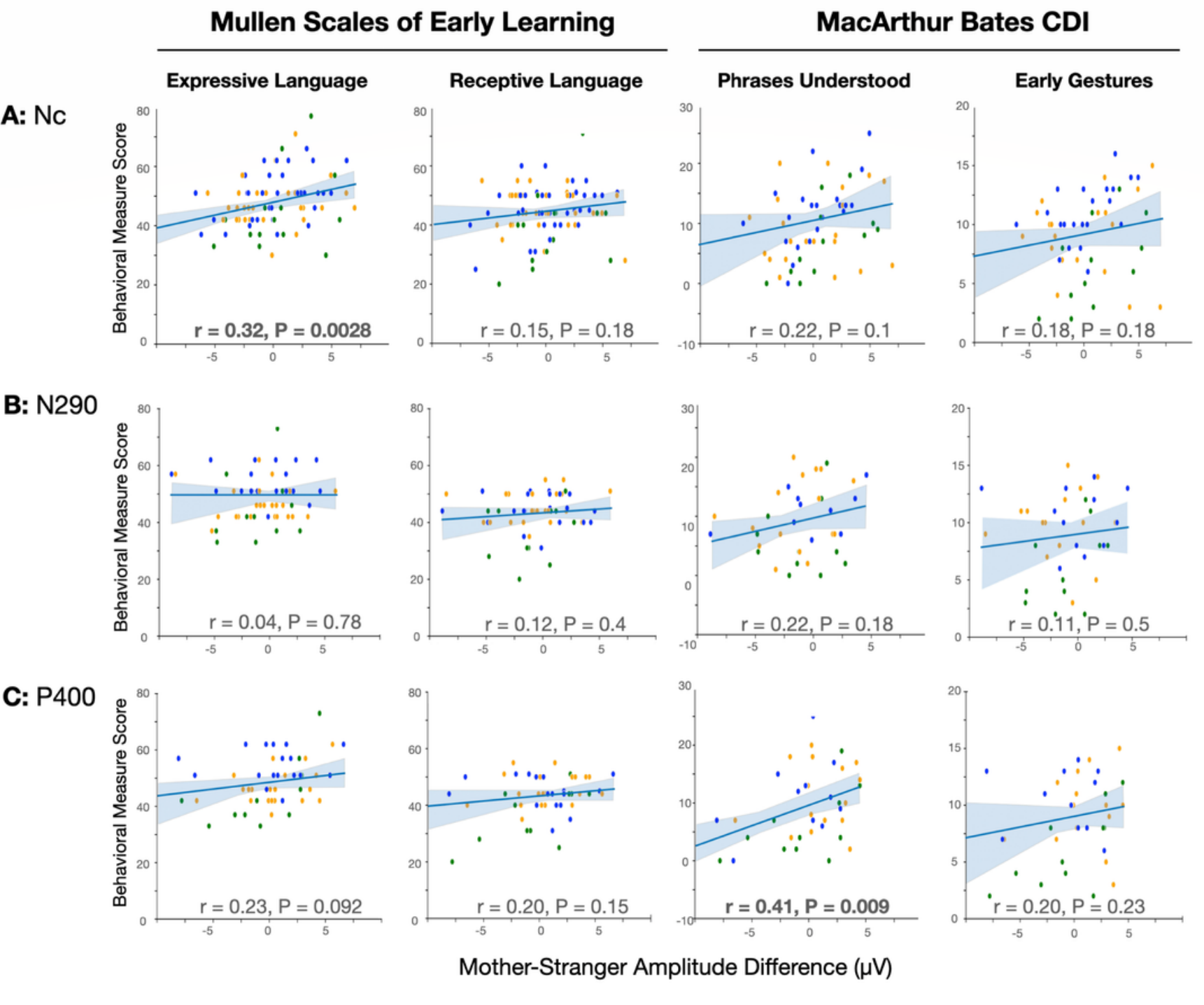

Figure 2

Mother-Stranger Amplitude Difference and Communication Measures. Correlations and Pearson $r$ statistics are shown between ERP amplitudes (A - Nc, B - N290, C P400) and the following 12-month communication measures: Mullen Scales of Early Learning Expressive Language and Receptive Language T-scores, MacArther Bates CDI Phrases Understood and Early Gestures raw scores. Blue $=$ LR, Orange $=$ HR-NoASD, Green $=$ HR-ASD 
ADOS Social Score

A: Nc

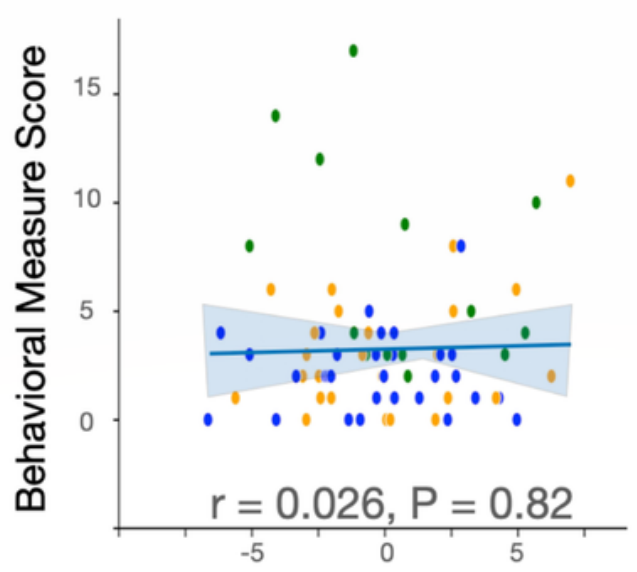

B: N290

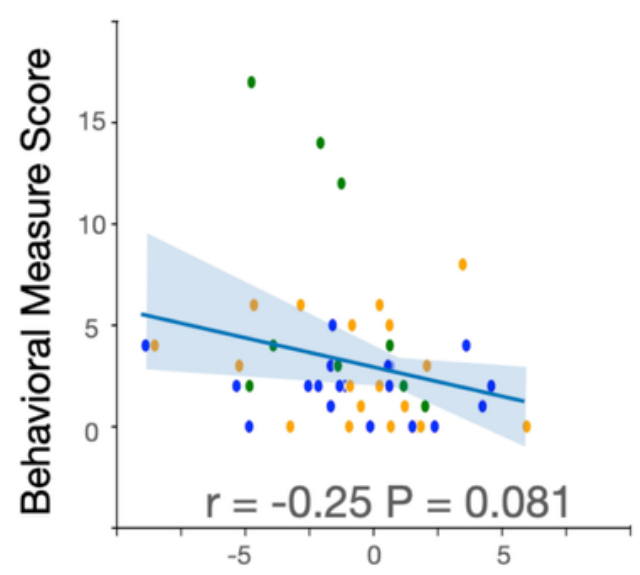

C: P400

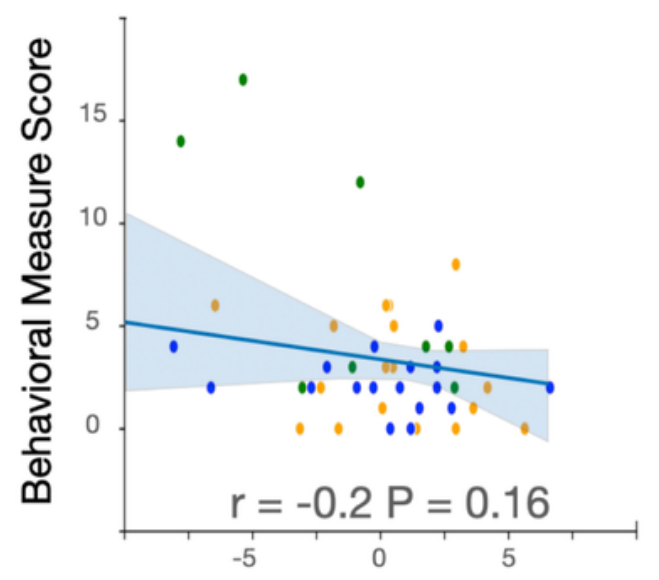

CSBS Social Score
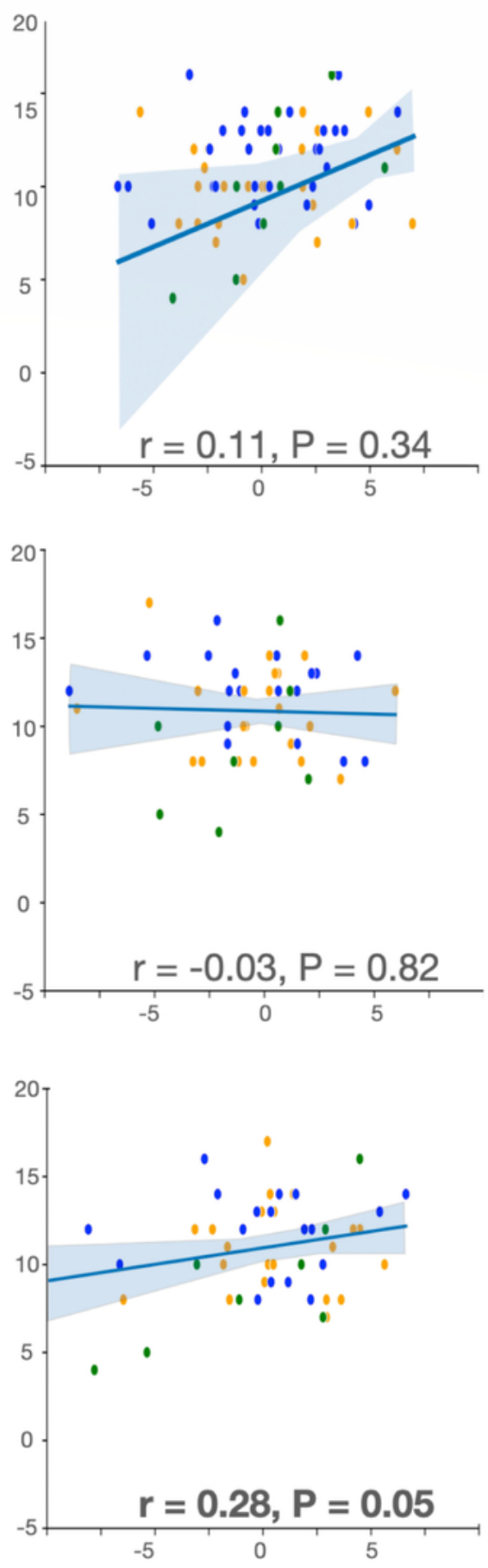

Mother-Stranger Amplitude Difference $(\mu \mathrm{V})$

Figure 3

Mother-Stranger Amplitude Difference and 18 month Social Measures. Correlations and Pearson $\mathrm{r}$ statistics are shown between ERP amplitudes (A - Nc, B - N290, C P400) and ADOS or CSBS Social Scores at 18 months. Blue $=\mathrm{LR}$, Orange $=\mathrm{HR}-$ NoASD, Green $=$ HR-ASD

\section{Supplementary Files}


This is a list of supplementary files associated with this preprint. Click to download.

- SupplementalFigures.pdf

- SupplementalTables.pdf 